\title{
Predictors of lymph node status in penile cancer: High-quality administrative datasets give us insight into what's happening in our own backyard
}

\author{
Luke T. Lavallée, MD \\ Urologic Oncology, Division of Urology, Ottawa Hospital Research Institute, The Ottawa Hospital and University of Ottawa, Ottawa, ON, Canada
}

Cite as: Can Urol Assoc J 2018;12(4):126. http://dx.doi.org/10.5489/cuaj.5278

See related article on page 119

$\mathrm{T}$ his population-based study from Ontario examined risk factors for positive lymph nodes (LN) in patients with squamous cell carcinoma (SCC) of the penis. ${ }^{1}$ Lower patient age, higher grade, and higher stage were independently associated with $\mathrm{LN}$ status in multivariable models. In addition, the study externally validated three risk stratification tools (European Association of Urology [EAU], International Consultation on Urological Diseases [ICUD], and National Comprehensive Cancer Network [NCCN]) that may be used by physicians to select patients for inguinal LN dissection, with each model having similar ability to discriminate risk.

Use of the Ontario Cancer Registry, an administrative population-based dataset, for these analyses provided several advantages to this study. The authors were able to identify over 400 cases of a rare disease over a 10-year period, making it one of the larger cohorts of penile cancer published. The results are also more likely to reflect real-world care compared to cohorts from referral centres with a specific interest in this disease process, making them more generalizable. Furthermore, because the Ontario Cancer Registry is regulated and has previously been shown to have high accuracy for identifying primary cancer diagnoses, it is likely the study captured a very high proportion of penile cancer cases diagnosed in the province during the study period, making it less prone to referral bias.

One important potential pitfall associated with use of administrative data is misclassification of cases or outcomes because investigators may rely on codes rather than direct chart review. The authors were able to overcome this drawback by directly reviewing pathology reports to confirm the diagnosis and procedure pathology. When direct review of important variables is not possible, an alternative approach would be to validate the administrative codes prior to using them. Without validation, errors associated with coding may significantly influence results. ${ }^{2}$

A second limitation of this study is its inability to easily determine clinical factors such as physical examination of $\mathrm{LN}$, radiological staging, and patient choices. These data are not available because administrative data are not specifically designed for research purposes the way disease-specific institutional datasets are.

Several findings reported in this study are noteworthy. There were only 419 unique cases of penile SCC identified in Ontario (population 13 million) over 10 years. Among these, only 74 patients received inguinal LN dissection, an average of 7-8 cases per year in the entire province. These numbers support centralization of care to high-volume surgeons for patients with these tumours, certainly those with any risk factor for $\mathrm{LN}$ spread.

The authors of this study should be congratulated for their diligent work. Canada houses many high-quality administrative datasets and I believe more studies such as these are needed. They provide powerful, generalizable data and are relatively inexpensive and rapid to perform with appropriate expertise. Perhaps more importantly, these data give us insight into what's happening in our own backyard.

Competing interests: Dr. Lavallée has participated in advisory board meetings for Ferring and Sanofi; and received a grant from Sanofi.

\section{References}

1. Qu XM, Siemens DR, Lovie AV, et al. Validation of predictors for lymph node status in penile cancer: Results from a population-based cohort. Can Urol Assoc J 2018;12:119-25. http://dx.doi. org/10.5489/cuaj.4711

2. van Walraven $C$, Austin P. Administrative database research has unique characteristics that can risk biased results. J Clin Epidemiol 2012;65:126-31. https://doi.org/10.1016/i.jclinepi.2011.08.002

Correspondence: Dr. Luke T. Lavallée, Urologic Oncology, Division of Urology, Ottawa Hospital Research Institute, The Ottawa Hospital and University of Ottawa, Ottawa, ON, Canada; lulavallee@toh.ca 